\title{
Nomograma de la dilatación del cervix en el parto
}

\author{
Dres.: Alvaro Velasco Ch.* Arturo Franco D. ${ }^{* *}$ y Fabio Reyes M. ${ }^{* * *}$
}

Los análisis estadísticos gráficos del progreso de la dilatación cervical durante el trabajo del perto han permitido disipar la ambigüedad e inexactitud existente respecto al progreso del trabajo de parto normal y patológico. Gracias al análisis de estas gráficas que miden la relación de tiempo y dilatación de cuello, podemos en una etapa temprana detectar cualquier retardo en el trabajo de parto.

Las primeras observaciones estadísticas fueron efectuadas por Friedman (1, 2), y analizadas también por Studd (3). En estos trabajos se elaboraron curvas de la dilatación del cuello relacionadas con el tiempo transcurrido del trabajo de parto que permitieron establecer dos fases de progreso de la dilatación que fueron denominadas, la fase de latencia y fase de aceleración máxima.

La fase de latencia se inicia en el comienzo del parto y perdura hasta el

* Profesor Asociado, Departamento de Gi. necología y Obstetricia. Universidad Nacional.

** Adjunto Ginecoobstetra. Hospital Materno Infantil.

*** Adjunto Ginecoobstetra. Hospital Materno Infantil. comienzo de la fase activa con una duración promedio de 8.6 horas en las primíparas y $5.3^{\prime}$ horas en las multíparas.

La fase de aceleración máxima se inicia en el punto en que se aumenta la rapidez de la dilatación hasta la iniciación del segundo período del parto $y$ tiene una duración de 4.9 horas en la primípara y 2.2 horas en la multípara.

Entre la fase de latencia y fase de aceleración máxima describen una fase de aceleración que representa el ángulo del cambio de aceleración. Igualmente entre el final de la etapa de acelaración máxima y la iniciación del período expulsivo describen una corta curva denominada fase desaceleración.

El presente trabajo se realizó con el objeto de establecer si la evolución de la dilatación en nuestras pacientes es igual al establecido por Friedman ya que Studd ha sugerido que podría haber alguna diferencia en diferentes grupos étnicos.

\section{MATERIAL Y METODOS}

El presente estudio se efectuó en pacientes del Instituto Materno Infantil de Bogotá durante el año de 1980 y realizado por personal de la Facultad de Medi- 


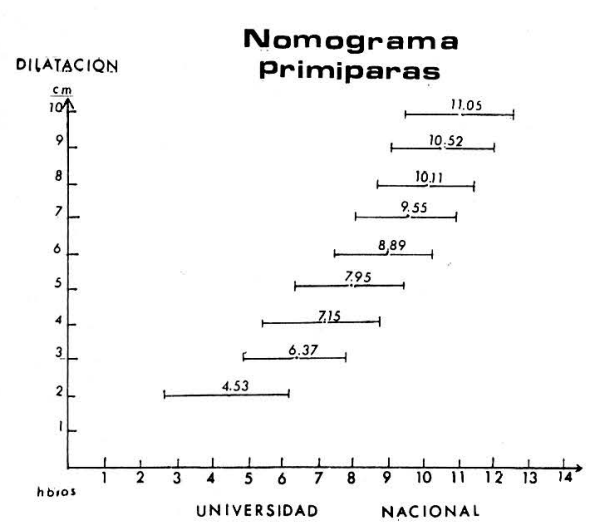

cina de la Universidad Nacional de Colombia.

Se analizaron 37 pacientes multíparas y 74 pacientes primíparas que reunieron las siguientes características; a) No recibieron ningún medicamento sedante, tocolítico, ni ocitócicos; b) No recibieron anestesia; c) El período expulsivo no fue intervenido; d) Las pacientes ingresaron durante la iniciación del trabajo de parto; e) Las membranas permanecieron integras hasta $8 \mathrm{cmts}$. de dilatación; f) La apreciación pélvica fue normal y el feto de tamaño proporcional; g) El recién nacido no ten ía sufrimiento fetal y nació con Apgar 10/10; h) El trabajo fue evaluado por lo menos con seis tactos vaginales para valorar la dilatación; i) La presentación debía ser de vértice; j) Embarazo a término; k) Clasificación de bajo riesgo.
Se consideró como iniciación del trabajo de parto la hora de ingreso al hospital siempre y cuando tuvieran menos de dos cmts. de dilatación en caso de primiparidad o tres en caso de multiparidad.

Los resultados obtenidos fueron analizados por métodos de promedios $v$ desviación estándar.

\section{RESULTADOS}

\section{Primigestantes}

Los resultados obtenidos pueden verse en la tabla No. 1 en donde se discrimina el tiempo por ctms. de dilatación.

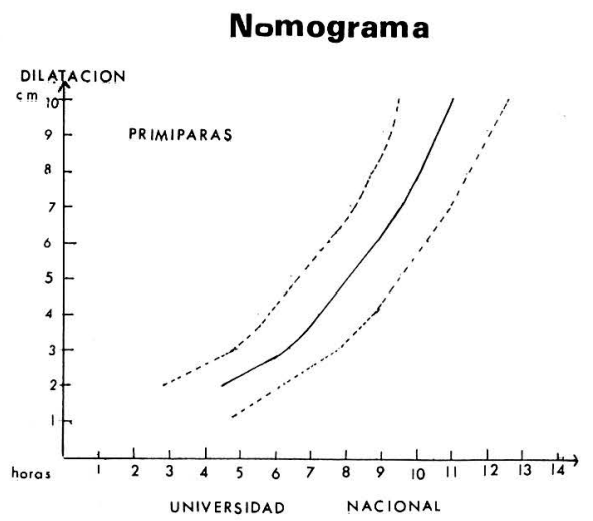

Se encontró que la duración total de la dilatación fue de 11 horas. La fase de

Tabla No. 1

DILATACION EN LAS PRIMIPARAS

\begin{tabular}{|lrrrrrrrrr|}
\hline Dilat. en Cms. & 2 & 3 & 4 & 5 & 6 & 7 & 8 & 9 & 10 \\
Promedio Hrs. & 4.53 & 6.37 & 7.15 & 7.95 & 8.89 & 9.55 & 10.11 & 10.52 & 11.85 \\
Desv. Estand. & 1.79 & 1.45 & 1.66 & 1.55 & 1.39 & 1.41 & 1.43 & 1.47 & 1.57 \\
\hline
\end{tabular}


latencia, incluyendo la pequeña curva de aceleración duró 7 horas y 6 minutos en promedio (DS $=1.66$ ), es decir $1 \mathrm{~cm}$., de dilatación en 1 hora y 45 minutos. La fase de aceleración máxima incluyendo la fase de desaceleración duró 3 horas y 54 minutos (DS $=1.57$ ) o sea $1 \mathrm{~cm}$. en 48 minutos.

La fase de aceleración máxima se inicia a los $4 \mathrm{cms}$. de dilatación.

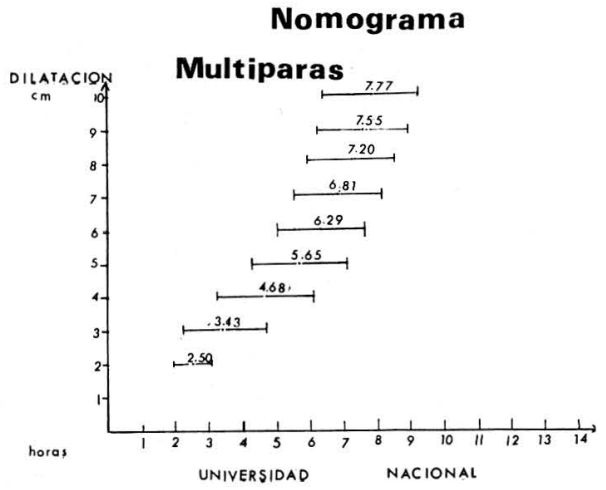

\section{MULTIPARAS}

Relacionado en la tabla No. 2, se encuentra la duración promedio según la dilatación del cuello en cmts.

La duración total del período de dilatación en las multíparas fue de $7 \mathrm{Hrs}$. y 46 minutos. La fase de latencia, en pro-
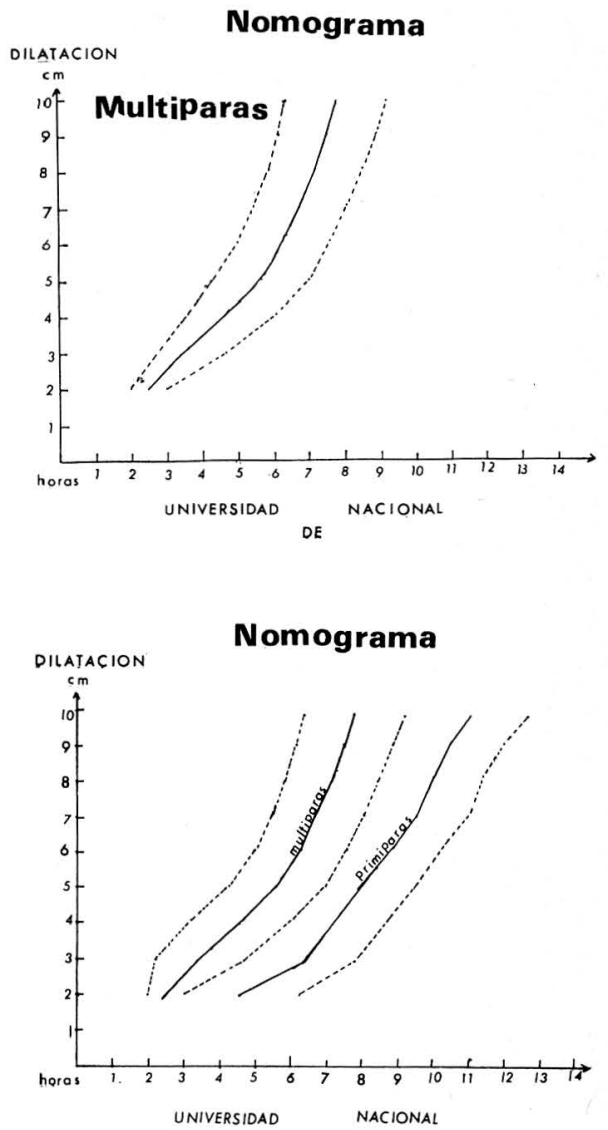

medio, incluyendo la fase de aceleración, fue de 5 horas y 39 minutos (DS = 1.45) lo que representa $1 \mathrm{~cm}$. por 1 hora.

Tabla No. 2

DILATACION EN LAS MULTIPARAS

\begin{tabular}{|lrrrrrrrrr|}
\hline Dilat. Cms. & 2 & 3 & 4 & 5 & 6 & 7 & 8 & 9 & 10 \\
Promedio Hrs. & 2.50 & 3.43 & 4.68 & 5.65 & 6.29 & 6.81 & 7.20 & 7.55 & 7.77 \\
Desv. Estand. & 0.50 & 1.24 & 1.45 & 1.37 & 1.28 & 1.31 & 1.30 & 1.35 & 1.40 \\
\hline
\end{tabular}


La fase de aceleración máxima fue de 2 horas y 7 minutos (DS $=1.40$ ) que equivale a $1 \mathrm{~cm}$. por $25 \mathrm{~min}$.

La fase de aceleración máxima se inicia a los $5 \mathrm{cms}$. de dilatación.

\section{COMENTARIOS}

Comparando los resultados obtenidos apreciamos que el trabajo total de las primigrávidas, 11 horas, es de una duración ligeramente más corta que la relatada por Friedman, 13 horas 30 minutos. Esta disminución se hace a expensas de una disminución en la fase de latencia.

En las multíparas no se apreció ninguna diferencia específica siendo ambas fases de igual duración.

En comparación con los promedios obtenidos en el trabajo de Studd, las cifras tanto de Friedman como las nuestras son significativamente más grandes; pero es de anotar que la mayoría de las pacientes estudiadas por este autor son analizadas a partir de un período más avanzado de dilatación.

Nuestras pacientes, debido a la gran selección que se hizo de ellas, pueden considerarse como ideales desde el punto de vista de un trabajo de parto normal; por esta razón consideramos que los datos obtenidos en nuestro estudio son de gran precisión.

El conocimiento íntimo del progreso de la dilatación permitirá al obstetra evaluar con exactitud la evolución nor- mal o anormal del trabajo de parto $y$ tomar las medidas pertinentes para la corrección oportuna de cualquier alteración existente. En esto se basan los diferentes partogramas existentes en diferentes fichas obstétricas.

Con frecuencia se observan estudios sobre el valor de los medicamentos aplicados durante el trabajo de parto en los cuales se presume que acorta el período de dilatación y relatan una aceleración de la dilatación como debida a la acción de la droga cuando ésta en realidad ha sido aplicada en la iniciación de la fase de aceleración en la cual hay una aceleración fisiológica de la aceleración.

\section{RESUMEN}

Se efectuó un trabajo para valorar el progreso de dilatación de cuello durante el trabajo de parto en 111 pacientes consideradas ideales desde todo punto de vista fisiológico. Treinta $\dot{y}$ siete pacientes multíparas y setenta y cuatro primíparas. Se elaboraron las curvas correspondientes y se compararon con las curvas obtenidas por otros autores. En las primíparas la dilatación del cuello duró 11 horas de las cuales 7 horas y 6 minutos correspondieron a la fase de latencia $y$ 3 horas y 54 minutos fueron de la fase de aceleración máxima. En las multíparas el período de dilatación duró 7 horas y 46 minutos de las cuales 5 horas y 39 minutos correspondieron a la fase de latencia y 2 horas y 7 minutos correspondieron a la fase de aceleración máxima.

\section{MONOGRAM OF CERVICAL DILATATION DURING DELIVERY}

\section{SUMMARY}

The purpose of this study was to assess progress in cervical dilatation during labor with 111 patients considered optimal from all physiological points of wue. Of these, 37 were multipara and 64 primipara. The appropriate graphs were drawn and compared with the findings of other authors. 
For the group of primipara, dilatation of the cervix took 11 hours, of which 7 hours and six minutes correspond to the latency phase and 3 hours and 54 minutes correspond to the phase of maximum acceleration. Among the multipara,

\section{BIBLIOGRAFIA}

1. FRIEDMAN, E.A. (1954). The Grafic Analysis of Labor, Am. J. of Obst. \& Gynaec. 68, 1568.

2. FRIEDMAN, E.A. (1955), Primigravid La- dilatation took 7 hours and 46 minutes, of which 5 hours and 39 minutes correspond to the latency phase and 2 hours and 7 minutes to the maximum acceleration phase. bor. A. Graphico-Estadistical Analysis, Obst. \& Gynaec. 6, 567.

3. STUDD, J. (1975). Control Gráfico del Trabajo de Parto, abril, 129. 